\title{
Advances in Diffusion-Weighted Imaging
}

\author{
Lorenzo Mannelli, MD, PhDa ${ }^{a}$ Stephanie Nougaret, MD ${ }^{a}, b$, Hebert A. Vargas, ${ }^{2}{ }^{a}$, and \\ Richard K. G. Do, MD, PhDa, ${ }^{\text {, }}$ \\ aDepartment of Radiology, Memorial Sloan Kettering Cancer Center, 1275 York Avenue, New \\ York, NY 10065, USA \\ bSt Eloi Hospital, CHU Montpellier, Montpellier, France
}

\section{Keywords}

Diffusion-weighted imaging; IVIM; Reproducibility; Apparent diffusion coefficient; MR imaging

\section{INTRODUCTION}

Diffusion-weighted imaging (DWI) has become an increasingly routine component of clinical MR imaging. Its unique soft tissue contrast mechanism exploits differences in the motion of water molecules in vivo at a biologically meaningful scale. The clinical potential of DWI in lesion detection, characterization, and response assessment has been explored in multiple organs and for multiple tumors. ${ }^{1,2}$ This review briefly covers basic principles of DWI and introduces some recent advances in the field, specifically for abdominopelvic organs. For additional introductory review articles, several excellent references are available. $^{3-5}$

\section{BASIC PRINCIPLES OF DIFFUSION-WEIGHTED IMAGING}

DWI is based on the use of single shot echoplanar imaging sequence with a long time to echo (TE) (60-100 ms), fat suppression, and the addition of motion-probing gradient pulses. When turned on, these gradients are used to decrease the signal intensity (SI) of moving water molecules during image acquisition. The SI of water molecules within each tissue decreases exponentially with the magnitude of their motion and with the strength of the motion-probing gradients (Equation 1).

$$
S_{b}=S_{0} \exp (-b \times A D C)
$$

In this monoexponential equation, the strength of the motion-probing gradients is summarized in a $b$ value that reflects the amplitude, duration, and interval between the

\footnotetext{
“Corresponding author: 1275 York Avenue, C-278D, New York, NY 10065. dok@mskcc.org. Disclosures: no relevant disclosures (L. Mannelli, S. Nougaret, and H.A. Vargas); Dr R.K.G. Do is a consultant/ advisor for Merck \& Co., Inc.
} 
gradients. The magnitude of the water diffusion is described by the apparent diffusion coefficient, or ADC, measured in $\mathrm{mm}^{2} / \mathrm{s} . S_{O}$ and $S_{b}$ are the baseline SI (before a motion probing-gradient is applied) and the SI at a prescribed $b$ value. Thus, various tissues lose their SI at different rates governed by their baseline SI, their ADC, and the choice of $b$ value.

\section{Intravoxel Incoherent Motion}

DWI performed in body imaging quickly revealed a non-monoexponential behavior of ADC as a function of the $b$ value. That is, the choice of $b$ value influenced the calculated ADC of tissue (Fig. 1). This observation was explained in part by work from Le Bihan and colleagues, ${ }^{6,7}$ recognizing that the motion of water molecules contributing to the signal in DWI arise from different compartments: extracellular space diffusion, intracellular space diffusion, and intravascular space diffusion (or perfusion). ${ }^{8}$ Separating the motion of water molecules owing to perfusion in the microcirculation from that owing to diffusion in the extravascular space is summarized by the methodology of intravoxel incoherent motion (IVIM) imaging (Equation 2).

$$
S_{b}=S_{0}\left[(1-f) \exp (-b \times D)+f \exp \left(-b \times D^{*}\right)\right]
$$

Where $S_{0}$ and $S_{b}$ represent the SI at baseline and at a specified $b$ value, $f$ represents the perfusion fraction (or the contribution of water moving in capillaries), $D$ represents the tissue diffusion coefficient, and $D^{*}$ represents the pseudodiffusion coefficient (or diffusion within the microcirculation). Because $D^{*}$ is greater than $D$ by several orders of magnitude, its contribution is negligible at higher $b$ values (typically above $b=200 \mathrm{~s} / \mathrm{mm}^{2}$ ). At higher $b$ values, the relationship between $S_{b}$ and $b$ again approximates a monoexponential equation dependent on $D$ (Fig. 2). This limitation led to the 2009 consensus statement on the use of DWI as an imaging biomarker, emphasizing the role of multiple $b$ values for measurement of $\mathrm{ADC}$, and the possibility of calculating $\mathrm{ADC}_{\text {high }}$, a surrogate for $D$, using only high $b$ value DWI. ${ }^{9}$

\section{Treatment Response}

Much excitement has been generated from the use of DWI in oncologic imaging because of its potential to monitor treatment response in vivo. ${ }^{4}$ The ability of DWI to evaluate oncologic outcomes can generally be separated into a pretreatment prediction of response, and the prediction of the response during or following treatment, such as chemotherapy or radiotherapy. The cellularity and vascularity of tumors are often affected by oncologic treatments, with potential changes affecting all 3 IVIM components, which are quantifiable by analysis of diffusion weighted images.

\section{Diffusion-Weighted Imaging Reproducibility}

Several barriers to the widespread adoption of DWI, especially in abdominal imaging, have included technical challenges to ensure a high-quality scan for every patient, and reproducible measurements of ADC. Quantifying the reproducibility of ADC and IVIM parameters is necessary before its use in clinical practice, and has been proposed by a panel 
of experts in the 2009 consensus statement. ${ }^{9}$ The challenges in generating reproducible ADC and IVIM parameters is highlighted in selected studies.

\section{ADVANCES IN LIVER DIFFUSION-WEIGHTED IMAGING}

In the last decade, the use of DWI in liver MR imaging has been focused on improving lesion detection, lesion characterization, and tumor response..$^{10-12}$ In addition, DWI has also been used to evaluate the liver parenchyma as a predictor of fibrosis and cirrhosis,,${ }^{13-17}$ a topic that is beyond the scope of this review. More recently, applications of IVIM in liver imaging and attempts at quantifying the reproducibility of DWI have been investigated.

\section{Intravoxel Incoherent Motion in Liver Lesion Characterization}

Distinguishing between benign and malignant liver lesion remains an important clinical challenge for radiologists. Initial studies with liver DWI were promising, showing differences in the ADC of benign and malignant lesions, although most studies included hepatic cysts and hemangiomas (with their corresponding high ADC values), entities that are often diagnosed easily with conventional T1 and T2 weighted imaging. The use of ADC to distinguish between solid benign and malignant liver lesions, such as hepatocellular carcinoma (HCC), focal nodular hyperplasia, or hepatocellular adenoma was not as promising. ${ }^{18}$

As multiple $b$ value DWI became available on clinical scanners, several groups investigated the potential of IVIM parameters for improved characterization of focal liver lesions. Similar to prior studies evaluating ADC of liver lesions, Ichikawa and colleagues ${ }^{19}$ found that $D$ was lower for malignant compared with benign lesions; however, it was not useful for distinguishing benign lesions that were not hemangiomas or cysts. Similarly, Yoon and colleagues ${ }^{20}$ compared the diagnostic performance of ADC and $D$ for distinguishing benign and malignant focal liver lesions in 142 patients. A higher area under the curve was found by receiving operating characteristic analysis for D compared with ADC, although this study also included 23 hemangiomas. Thus, the diagnostic utility of the IVIM parameter $D$ in characterizing a solid hypervascular liver lesion as malignant or benign remains uncertain.

Several studies have explored the utility of IVIM in further characterizing specific liver malignancies. The correlation between the ADC and $D$ of $\mathrm{HCC}$ with its histologic grade was investigated, demonstrating a higher accuracy for distinguishing high-grade from low-grade HCC by $D$ compared with ADC by receiving operating characteristic analysis. A stronger correlation between HCC tumor grade and $D(r=-0.604)$ was found compared with ADC $(r$ $=-0.448)$. The relatively modest sensitivity $(88.9 \%)$ and specificity $(78.3 \%)$ in distinguishing low-grade and high-grade HCC suggest that further refinement in IVIM technique and analysis, or combination with other MR imaging parameter, is needed before clinical application. For patients with colorectal cancer liver metastases on chemotherapy, both ADC and $D$ values on whole tumor region of interest (ROI) were correlated with percent tumor necrosis on pathology. ${ }^{21}$ On the other hand, a lack of correlation between necrosis with $D^{*}$ and $f$ was attributed to the general difficulty in fitting these parameters in the liver. 


\section{Reproducibility in the Liver}

Despite the advances obtained from IVIM over single $b$ value ADC measurements, the optimal DWI technique for clinical liver imaging remains unclear. The liver also poses additional challenges compared with other organs, because of its proximity to the diaphragm and heart, with related respiratory and cardiac motions. Several recent studies have investigated the reproducibility of DWI measurements, evaluating the impact of different $b$ values, as well as respiratory schemes, including breath hold (BH), free breathing (FB), respiratory triggering (RT), and navigator triggering (NT). ${ }^{22-24}$

For focal liver lesions, Choi and colleagues ${ }^{22}$ investigated potential differences in ADC measurements using BH, RT, and FB techniques. For focal liver lesions, excellent agreement in ADC (intraclass correlation $=0.952$ ) were found at $3.0 \mathrm{~T}$. In contrast, $\mathrm{ADC}$ of liver parenchyma had lower reproducibility with BH versus FB, RT, and NT respiratory techniques, although the results were possibly because of the lower number of excitations $\left(\mathrm{NE}_{\mathrm{X}}\right)$ used for $\mathrm{BH}\left(\mathrm{NE}_{\mathrm{X}}=2\right)$ versus other techniques $\left(\mathrm{NE}_{\mathrm{X}}=4\right) .{ }^{24}$ Chen and colleagues ${ }^{24}$ also found lesser $\mathrm{ADC}$ reproducibility and greater ADC mean values for the left compared with the right hepatic lobe, and decreasing ADC values as one measures from the superior to inferior left hepatic lobe, findings that reflect the potential effects of cardiac motion. These results further reinforce the suggestion by the consensus statement on DWI to measure ADC of focal liver lesions only in the right hepatic lobe. ${ }^{9}$ Based on the limits of agreement for ADC measurements, the authors recommend a threshold of a $30 \%$ difference when using $\mathrm{ADC}$ as a biomarker, on baseline and follow-up studies. In a study of 20 patients with liver metastases, the potential use of ADC was investigated to predict treatment response to chemotherapy. ${ }^{25}$ A similar repeatability coefficient of plus or minus $28 \%$ was found for $\mathrm{ADC}$ of liver metastases when limited to right hepatic lobe.

The choice of $b$ values can influence the reproducibility of ADC measurements across various respiratory techniques, as demonstrated in a study that included 10 volunteers and 11 patients with focal liver lesions. ${ }^{26}$ For example, higher maximal $b$ values included in the ADC calculations led to higher reproducibility for FB and RT techniques. This study further highlights the difficulty in comparing the reproducibility of ADC measurements across various studies using different acquisition techniques and methods of ADC analysis. Future studies will need to demonstrate consistent DWI technique when using ADC as a biomarker for treatment response of liver malignancies.

The reproducibility of IVIM parameters for liver DWI has been more challenging, as illustrated in several recent works. There was good to excellent reproducibility for $D$, but moderate to good and poor to moderate reproducibility for $f$ and $D^{*}$, respectively, in a prospective study evaluating FB and RT DWI in volunteers and patients for evaluation of liver fibrosis. ${ }^{23}$ Similarly, there was poor reproducibility of $f$ and $D^{*}$, and good reproducibility for $D$ and $A D C$ for $\mathrm{HCC}$ in a study of 10 patients with DWI performed on 2 separate MR imagings. ${ }^{27}$ Despite the excitement surrounding IVIM in liver MR imaging, refinements in this technique and greater consensus on optimal techniques for clinical sites are needed to ascertain a baseline reproducibility in IVIM parameters. 
At our own institution, we routinely use a BH DWI technique with multiple $b$ values, and for patients with limited BH capacity, NT is available. With BH techniques, acquisition times are limited to 20 to 25 seconds, with the $\mathrm{NE}_{\mathrm{x}}$ consequently limited to 2 . Higher $\mathrm{NE}_{\mathrm{x}}$ are possible with NT, but with longer resulting acquisition times (Table 1).

\section{ADVANCES IN PANCREAS DIFFUSION-WEIGHTED IMAGING}

Improvements in DWI techniques have also advanced the potential clinical applications in pancreatic imaging. Several studies are available on pancreatic lesion detection ${ }^{28,29}$ and characterization, ${ }^{30-32}$ normal pancreatic parenchyma, ${ }^{33,34}$ and pancreatic function. ${ }^{35,36}$

Pancreatic tumors can be categorized into 4 main groups: cystic tumors, neuroendocrine tumors (NETs), solid nonendocrine neoplasms, and pseudotumors (chronic or acute inflammatory changes). Some of these lesions have overlapping clinical presentations and imaging is often requested to detect, characterize, and eventually stage these lesions. Some of the common clinical questions include how to differentiate indolent from malignant NETs, how to distinguish between tumors and pseudotumors, and how to identify benign from malignant cystic tumors. The potential utility of DWI and IVIM has been explored to address these clinical challenges. ${ }^{28-32,37-47}$

\section{Diffusion-Weighted Imaging in Pancreatic Lesion Detection and Characterization}

Normal pancreatic parenchyma is characterized by high ADC values with a mild gradient from the head toward the tail. ${ }^{33,44}$ Noncystic pancreatic tumors demonstrate typically lower ADC values than normal parenchyma, and have higher SI compared with adjacent pancreatic parenchyma on DWI, making this technique a potentially useful tool for pancreatic tumor detection.

The relationship between tumor ADC and histologic grade has been explored for both cystic and solid pancreatic tumors. Two studies have investigated the potential prognostic significance of ADC values for pancreatic NETs. In the first, an inverse correlation was found between the cellular marker for proliferation Ki-67 and ADC values, that is, a lower $\mathrm{ADC}$ would indicate a more rapidly proliferating tumor, ${ }^{39}$ whereas in the second study, when an ADC ratio between NETs and adjacent pancreatic parenchyma was calculated, a cutoff value of 1.03 could discriminate between benign and nonbenign NETs with a sensitivity of $92.9 \%$ and a specificity of $84.6 \% .^{31}$

Conflicting data have been published on the correlation between pancreatic adenocarcinoma pathologic grade and ADC values. Wang and colleagues ${ }^{48}$ and Hayano and colleagues ${ }^{49}$ demonstrated that the tumor ADC values correlate with their pathologic grade of differentiation: high-grade adenocarcinomas with limited glandular formation and dense fibrosis had lower ADC values than low-grade adenocarcinomas. However, Rosenkrantz and colleagues $^{50}$ found no such a correlation in 30 patients with adenocarcinoma. Significant differences exist between these studies with respect to the DWI sequences, $b$ values, ROI placement, and type of ADC analysis. For example, Hayano and colleagues used minimum ADC values, whereas the other 2 studies used mean ADC values. In addition, Rosenkrantz and colleagues included the whole lesion in the ROI and the other 2 studies included only 
the solid and homogeneous portions of the tumor. The variability in how similar DWI datasets can be analyzed remains an obstacle for the widespread adoption of this technique for clinical use.

Differentiating between benign and malignant intraductal papillary mucinous neoplasms (IPMN) remains challenging for clinicians who are increasingly following patients with cystic pancreatic lesions. Although a consensus statement is available to guide the selection of IPMN for surgery, the guidelines remain imperfect. ${ }^{51,52}$ In a cohort of 52 patients with cystic IPMN, Kang and colleagues ${ }^{53}$ demonstrated lower ADC in malignant IPMN compared with benign IPMN. In this study, adding DWI to conventional MR cholangiopancreatography, 3 observers were able to differentiate between benign and malignant IPMN with a sensitivity ranging between $76 \%$ and $100 \%$ and a specificity between $84 \%$ and $97 \%$. In a group of 50 patients, Fatima and colleagues ${ }^{54}$ were able to define a $2.4 \times 10^{-3} \mathrm{~mm}^{2} / \mathrm{s}$ cutoff ADC value to differentiate between IPMN and MCN with a sensitivity of $98 \%$ and a specificity of $88 \%$. Schraibman and colleagues ${ }^{38}$ compared ADC values of serous cystadenoma and mucinous cystic tumors in 45 subjects and showed that mucinous tumors have lower ADCs. They proposed a greater restricted diffusion of water in the presence of mucin. Whether ADC measurements may be useful in cystic pancreatic lesions in a prospective setting remains to be seen.

\section{Intravoxel Incoherent Motion in Pancreatic Lesion Characterization}

At this time, only 4 groups have evaluated IVIM in pancreas imaging. ${ }^{30,43-45,55,56}$ The evidence thus far suggests that normal pancreatic tissue demonstrates higher IVIM parameters $D$ and $f$ than non-cystic pancreatic lesions. In a group of 71 patients, Lee and colleagues ${ }^{45}$ also reported $87.2 \%$ sensitivity and $61.5 \%$ specificity when using $D$ to differentiate pseudotumors from solid nonendocrine neoplasms. In the same study, solid nonendocrine neoplasms had significantly higher $f$ compared with pseudotumors, and $f$ could be used with a $92.3 \%$ specificity and $42.6 \%$ sensitivity in differentiating the same entities using $23.91 \%$ as cutoff value. In a smaller group of patients, Concia and colleagues ${ }^{44}$ confirmed significant $f$ values differences between neoplastic lesions and pseudotumors, but reported overlap of $D$ values between the same entities. Kang and colleagues $^{30}$ in a group of 46 patients also demonstrated significantly lower $f$ values in neoplastic lesions compared with pseudotumors with a sensitivity of $84 \%$ and a specificity of $100 \%$ in differentiating these 2 entities. In the same study, the authors analyzed fast and slow water molecules diffusion and found that no differences in $D$ between pseudotumors and neoplastic lesions; however, it was possible to differentiate these 2 pathologic processes using $D^{*}$ measurements with a sensitivity of $94.9 \%$ and a specificity of $85.7 \%$. In 37 patients with IPMN, Kang and colleagues were able differentiate between benign and malignant IPMN analyzing $D$ and $f$ values of the solid portions of these lesions. With $f$, a sensitivity of $93.3 \%$ and a specificity of $77.3 \%$ was achieved in differentiating benign versus malignant IPMN, whereas slow $D$ had a sensitivity of $93.3 \%$ and a specificity $63.6 \%$. No differences were found between these 2 entities when using analyzing $D^{*}$ values.

In summary, the initial experiences with IVIM for pancreatic pathologies are promising in their potential to discriminate between carcinoma of the pancreas and chronic pancreatitis, 
and between malignant and benign IPMN. However, the pulse sequences and methods for quantitative analysis differ between the different experiments and further studies are needed to validate these results.

\section{Reproducibility in the Pancreas}

Few studies have investigated the reproducibility of DWI for the pancreas. Ye and colleagues ${ }^{57}$ found negligible $(<5 \%)$ differences in ADC measurements of the pancreas in 24 healthy volunteers; these subjects were imaged during 2 different session using 2 different 1.5-T scanners and a 3-T scanner. No differences in normal pancreas ADC values were found before and after injection of intravenous gadolinium contrast agents ${ }^{58}$; similar results were demonstrated in pancreatic ductal adenocarcinoma. ${ }^{59}$ The lack of differences in ADC before and after contrast should allow for some flexibility in pancreas MR protocol design, with the implementation of DWI sequences before or after contrast administration. Herrmann and colleagues ${ }^{34}$ reported ADC values differences related to subjects age and sex; this observation may affect the use of ADC ratios to normal pancreas when characterizing focal pancreatic lesions. To date, no reproducibility study on IVIM parameters in pancreas DWI have been published.

At our own institution, during MR cholangiopan-creatography studies, we currently perform both large field of view (FOV) DWI, as done for the liver DWI, and a small FOV DWI. Small, or reduced, FOV DWI is a recently available technique on GE scanners that allows for higher resolution imaging with reduced artifacts (Fig. 3). With the smaller voxel size, however, NT is required to achieve sufficient signal to noise (see Table 1).

\section{ADVANCES IN RECTAL DIFFUSION-WEIGHTED IMAGING}

Dramatic advances in image quality over the past few years have made DWI a promising tool for rectal lesion evaluation. As such, recent guidelines for rectal cancer imaging recommend DWI as part of a standard MR imaging protocol for preoperative restaging of rectal cancers after neoadjuvant chemoradiotherapy (CRT) ${ }^{60}$

\section{Diffusion-Weighted Imaging for Initial Evaluation: Rectal Cancer Detection, Characterization, and Staging}

Rectal cancer detection relies on clinical examination and endoscopy. However, in some cases DWI helps to locate very small rectal tumors during primary staging. ${ }^{61}$ For characterization, several studies have demonstrated lower ADC values in rectal adenocarcinoma compared with normal rectal wall. ${ }^{61}$ Curvo-Semedo and colleagues ${ }^{62}$ also reported lower ADC values in more aggressive tumors. The added value of DWI for primary rectal cancer staging is still debated. DWI can facilitate lymph node detection, but alone it is not reliable for differentiating between benign and malignant lymph nodes. ${ }^{63}$

\section{Evaluating the Response to Neoadjuvant Treatment with Diffusion-Weighted Imaging}

The treatment of locally advanced rectal cancer has shifted in recent years from a standard treatment for all patients including neoadjuvant CRT followed by operative resection to a more conservative approach. Response evaluation of locally advanced rectal cancer after 
CRT is thus emerging as a critical issue. Recent studies have illustrated the added value of DWI to differentiate viable tumor from fibrosis and to allow prediction of complete response. ${ }^{64-70}$ Areas of fibrosis typically have a low cellular density, which results in low SI on high $b$ value DWI. In contrast, residual tumor areas have a relatively high cellular density and show high SI on DWI images that stands out against the low SI of the surrounding tissue and fibrosis. As such, small areas of residual tumor are better depicted on DWI images (Fig. 4). More recently, tumor volumetry with DWI has demonstrated highly accurate results in prediction of complete tumor response. ${ }^{70}$

The results from studies evaluating pre-CRT ADC values to predict rectal tumor response to neoadjuvant treatment are somewhat conflicting. Some authors have suggested that initial $\mathrm{ADC}$ values might predict tumor response to $\mathrm{CRT}^{71-73}$ and others have demonstrated significantly lower pre-CRT ADC values in good responders compared with poor responders. ${ }^{64,71}$ Conversely, other authors found no difference in the pre-CRT distribution of ADC values between nonresponders and responders. ${ }^{70,74,75}$ Likewise, results concerning post CRT tumor response lack agreement: several studies have demonstrated significantly higher mean post-treatment ADC values in the complete responder group, ${ }^{66,74,76}$ whereas others reported no difference. ${ }^{70}$

\section{Reproducibility in the Rectum}

ADC values often show great variability and are difficult to compare owing to differences in methodology. Most studies with ADC measurements have been conducted on selected ROIs placed on a representative image of the rectal tumor for analysis. ${ }^{64,74,76,77}$ Thus, these techniques do not assess the problem of variable ROI size and positioning. In a study performed by Lambregts and colleagues, ${ }^{78}$ the authors found that ADCs acquired from the whole tumor volume were more reproducible than those obtained from single-slice or small sample measurements.

Although normalized ADC values have been suggested, in pancreas ADC, for example, no similar studies have ever been performed in rectal DWI. ${ }^{79}$ One study analyzed the repeatability of rectal tumor $\mathrm{ADC}$ at $1.5 \mathrm{~T} .{ }^{80}$ In this study, 18 patients with rectal cancer underwent a rectal MR imaging on 5 consecutive days with 2 identical DWI sequences. The repeatability coefficient of the ADC value was $9.8 \%$, which is in the range of previous reported study of ADC value in the abdomen. ${ }^{80}$

\section{Intravoxel Incoherent Motion in Rectal Cancer}

To date, only preliminary results are available from a single study on the use of IVIM for rectal cancer. Bauerle and colleagues ${ }^{81}$ compared the IVIM parameters $(f$ and $D)$ with histologic data (vascular area fraction and vessel diameter). First, although $f$ was found to be significantly higher in the normal rectal wall of patients than in the tumor, histologic results showed significantly higher vascular area fractions in the tumor than in the rectum. The authors attributed their findings to the irregular morphology of tumor vasculature that led to a moderate perfusion in small and tortuous vessels. This paradoxic result has already been observed in some other tissues, such as prostate cancers versus surrounding normal tissues ${ }^{39}$ 
and lung cancer versus consolidation. ${ }^{40}$ Moreover in this study, the parameters $D$ and $f$ were correlated with the vascular area fraction in patients without CRT.

The negative correlation found between $D$ and the vascular area fraction in these patients could be an indicator that $D$ is associated with tumor tissue, which is often strongly vascularized. In contradistinction, in patients who received CRT, the absence of correlations in rectal carcinoma between DWI and histologic parameters suggests that therapy induced inhomogeneous changes, such as focal fibrosis and necrosis, as well as changes in vessel permeability. Further studies are needed to validate prospectively the utility of IVIM parameters such as $D$, as a noninvasive imaging biomarker in rectal cancer.

At our own institution, we routinely use BH DWI technique with multiple $b$ values (see Table 1). It is critical that the DWI sequence matches the oblique T2W axial sequence perpendicular to the tumor to assess correctly tumor response and to perform fused imaging.

\section{ADVANCES IN PROSTATE DIFFUSION-WEIGHTED IMAGING}

The use of DWI in prostate cancer has been the subject of extensive research in the literature and is now included most clinical prostate MR imaging protocols. Simple DWI-derived quantitative metrics such as the ADC have been used to differentiate benign and malignant prostate tissue. ${ }^{82}$ Also, significant inverse correlations have been reported between ADC values and prostate cancer Gleason score as well as tumor proliferation markers such as Ki-67. ${ }^{82-84}$ Despite these significant differences, substantial overlap exists between the ADC values of prostate cancer and benign conditions such as prostatitis and biopsy-induced changes. As a result, recent efforts have focused on more advanced methods for DWI acquisition, processing, and interpretation, with the aim of consolidating and further developing its well-established role in prostate cancer assessment.

\section{Choice of b Values in Prostate Diffusion-Weighted Imaging}

The optimal choice and number of $b$ values that should be used to acquire prostate DWI is a matter of continuous debate. Absolute ADC values strongly depend on the choice of $b$ values and therefore should be used with caution, particularly when attempting to establish "thresholds" for diagnosing disease states. ${ }^{85}$ Higher $b$ values offer the theoretic advantage of increased tumor to normal tissue contrast, at the expense of a decreased signal-to-noise ratio. One study reported higher lesion conspicuity and tumor-normal SI ratio when DWI was acquired with $b$ values of 0 and $2000 \mathrm{~s} / \mathrm{mm}^{2}$ compared with 0 and $1000 \mathrm{~s} / \mathrm{mm}^{2.86}$ Others suggest that, rather than acquiring high $b$ value images, these can be "computed" by voxelwise fitting from a set of acquired lower $b$ value images. Using numerical simulations, Maas and colleagues ${ }^{86}$ reported comparable noise and the contrast-to-noise ratios between "calculated" and "acquired" DWI at a $b$ value of $1400 \mathrm{~s} / \mathrm{mm}^{2}(P=.395)$. Another study reported the best diagnostic performance for prostate tumor detection when $\mathrm{T} 2$ weighted images were evaluated in conjunction with b2000 DWI, regardless of whether a set of "measured" or "calculated" b2000 images were used. ${ }^{87}$ 


\section{Intravoxel Incoherent Motion in Prostate Cancer}

The number of $b$ values used in prostate DWI is also controversial. A minimum of $2 b$ values are required for monoexponential calculation of ADC. The use of 3 or more $b$ values can better account for the non-monoexponential behavior of ADC as a function of $b$ value and the influence of perfusion at low $b$ values. There is evidence that IVIM parameters, including $D, D^{*}$ and $f$, differ significantly between cancerous and normal peripheral zone prostatic tissue cancer. ${ }^{88,89}$ Others have questioned the incremental value IVIM over simple monoexponential ADC measurements in prostate cancer, with some issues being raised about the influence of the $b$ values used for acquisition and ideal method for calculating IVIM parameters. ${ }^{90,91}$ One of these studies found significant differences in IVIM parameters using 2 different methods for calculation. ${ }^{90}$ Despite this, the IVIM parameter $D$ (but not $f$ or $\left.D^{*}\right)$ was able to discriminate between tumor and normal areas with an area under the curve of 0.90 or greater in this study, regardless of the calculation method..$^{90}$ Further research into prostate IVIM is needed, with a focus on standardization of image acquisition techniques and approaches to fit the IVIM parameters from the measured DWI data.

At our own institution, we use a non-BH DWI technique with multiple $b$ values (b 0, 400, $700,1000)$. As described for the pancreas, we also use large and reduced FOV DWI for prostate imaging, which allows for higher resolution imaging with decreased artifacts. A summary of the DWI acquisition parameters is shown in Table 1.

\section{SUMMARY}

Exciting developments in DWI, both in imaging techniques and applications, have improved our ability to detect and characterize benign and malignant neoplasms. Predicting the histologic grade of malignancies and their response to treatment continue to be active areas of research. Multiple challenges remain, such as the standardization of acquisition parameters and DWI analysis across sites, to further the adoption of advanced methodologies, such as IVIM, to clinical practice.

\section{References}

1. Moore WA, Khatri G, Madhuranthakam AJ, et al. Added value of diffusion-weighted acquisitions in MRI of the abdomen and pelvis. AJR Am J Roentgenol. 2014; 202(5):995-1006. [PubMed: 24758652]

2. Rosenkrantz AB, Oei M, Babb JS, et al. Diffusion-weighted imaging of the abdomen at 3.0 Tesla: image quality and apparent diffusion coefficient reproducibility compared with 1. 5 Tesla. J Magn Reson Imaging. 2011; 33(1):128-35. [PubMed: 21182130]

3. Bonekamp S, Corona-Villalobos CP, Kamel IR. Oncologic applications of diffusion-weighted MRI in the body. J Magn Reson Imaging. 2012; 35(2):257-79. [PubMed: 22271274]

4. Koh DM, Collins DJ. Diffusion-weighted MRI in the body: applications and challenges in oncology. AJR Am J Roentgenol. 2007; 188(6):1622-35. [PubMed: 17515386]

5. Qayyum A. Diffusion-weighted imaging in the abdomen and pelvis: concepts and applications. Radiographics. 2009; 29(6):1797-810. [PubMed: 19959522]

6. Le Bihan D, Breton E, Lallemand D, et al. Separation of diffusion and perfusion in intravoxel incoherent motion MR imaging. Radiology. 1988; 168(2):497-505. [PubMed: 3393671]

7. Le Bihan D, Breton E, Lallemand D, et al. MR imaging of intravoxel incoherent motions: application to diffusion and perfusion in neurologic disorders. Radiology. 1986; 161(2):401-7. [PubMed: 3763909] 
8. Dixon WT. Separation of diffusion and perfusion in intravoxel incoherent motion MR imaging: a modest proposal with tremendous potential. Radiology. 1988; 168(2):566-7. [PubMed: 3393682]

9. Padhani AR, Liu G, Koh DM, et al. Diffusion-weighted magnetic resonance imaging as a cancer biomarker: consensus and recommendations. Neoplasia. 2009; 11(2):102-25. [PubMed: 19186405]

10. Coenegrachts K, Delanote J, Ter Beek L, et al. Improved focal liver lesion detection: comparison of single-shot diffusion-weighted echoplanar and single-shot T2 weighted turbo spin echo techniques. Br J Radiol. 2007; 80(955):524-31. [PubMed: 17510250]

11. Galea N, Cantisani V, Taouli B. Liver lesion detection and characterization: role of diffusionweighted imaging. J Magn Reson Imaging. 2013; 37(6):1260-76. [PubMed: 23712841]

12. Parikh T, Drew SJ, Lee VS, et al. Focal liver lesion detection and characterization with diffusionweighted MR imaging: comparison with standard breath-hold T2-weighted imaging. Radiology. 2008; 246(3):812-22. [PubMed: 18223123]

13. Do RK, Chandarana H, Felker E, et al. Diagnosis of liver fibrosis and cirrhosis with diffusionweighted imaging: value of normalized apparent diffusion coefficient using the spleen as reference organ. AJR Am J Roentgenol. 2010; 195(3):671-6. [PubMed: 20729445]

14. Girometti R, Furlan A, Esposito G, et al. Relevance of b-values in evaluating liver fibrosis: a study in healthy and cirrhotic subjects using two single-shot spin-echo echo-planar diffusion-weighted sequences. J Magn Reson Imaging. 2008; 28(2):411-9. [PubMed: 18666139]

15. Razek AA, Khashaba M, Abdalla A, et al. Apparent diffusion coefficient value of hepatic fibrosis and inflammation in children with chronic hepatitis. Radiol Med. 2014; 119:903-9. [PubMed: 24846081]

16. Taouli B, Chouli M, Martin AJ, et al. Chronic hepatitis: role of diffusion-weighted imaging and diffusion tensor imaging for the diagnosis of liver fibrosis and inflammation. J Magn Reson Imaging. 2008; 28(1):89-95. [PubMed: 18581382]

17. Chandarana H, Do RK, Mussi TC, et al. The effect of liver iron deposition on hepatic apparent diffusion coefficient values in cirrhosis. AJR Am J Roentgenol. 2012; 199(4):803-8. [PubMed: 22997371]

18. Taouli B. Diffusion-weighted MR imaging for liver lesion characterization: a critical look. Radiology. 2012; 262(2):378-80. [PubMed: 22282176]

19. Ichikawa S, Motosugi U, Ichikawa T, et al. Intravoxel incoherent motion imaging of focal hepatic lesions. J Magn Reson Imaging. 2013; 37(6):1371-6. [PubMed: 23172819]

20. Yoon JH, Lee JM, Yu MH, et al. Evaluation of hepatic focal lesions using diffusion-weighted MR imaging: comparison of apparent diffusion coefficient and intravoxel incoherent motion-derived parameters. J Magn Reson Imaging. 2014; 39(2):276-85. [PubMed: 23633178]

21. Chiaradia M, Baranes L, Van Nhieu JT, et al. Intravoxel incoherent motion (IVIM) MR imaging of colorectal liver metastases: are we only looking at tumor necrosis? J Magn Reson Imaging. 2014; 39(2):317-25. [PubMed: 23723012]

22. Choi JS, Kim MJ, Chung YE, et al. Comparison of breathhold, navigator-triggered, and freebreathing diffusion-weighted MRI for focal hepatic lesions. J Magn Reson Imaging. 2013; 38(1): 109-18. [PubMed: 23188562]

23. Dyvorne HA, Galea N, Nevers T, et al. Diffusion-weighted imaging of the liver with multiple b values: effect of diffusion gradient polarity and breathing acquisition on image quality and intravoxel incoherent motion parameters-a pilot study. Radiology. 2013; 266(3):920-9. [PubMed: 23220895]

24. Chen X, Qin L, Pan D, et al. Liver diffusion-weighted MR imaging: reproducibility comparison of ADC measurements obtained with multiple breath-hold, free-breathing, respiratory-triggered, and navigator-triggered techniques. Radiology. 2014; 271(1):113-25. [PubMed: 24475860]

25. Deckers F, De Foer B, Van Mieghem F, et al. Apparent diffusion coefficient measurements as very early predictive markers of response to chemotherapy in hepatic metastasis: a preliminary investigation of reproducibility and diagnostic value. J Magn Reson Imaging. 2014; 40:448-56. [PubMed: 24924334]

26. Larsen NE, Haack S, Larsen LP, et al. Quantitative liver ADC measurements using diffusionweighted MRI at 3 Tesla: evaluation of reproducibility and perfusion dependence using different techniques for respiratory compensation. MAGMA. 2013; 26(5):431-42. [PubMed: 23483359] 
27. Kakite S, Dyvorne H, Besa C, et al. Hepatocellular carcinoma: short-term reproducibility of apparent diffusion coefficient and intravoxel incoherent motion parameters at 3.0T. J Magn Reson Imaging. 2015; 41:149-56. [PubMed: 24415565]

28. Wiggermann P, Grutzmann R, Weissenbock A, et al. Apparent diffusion coefficient measurements of the pancreas, pancreas carcinoma, and mass-forming focal pancreatitis. Acta Radiol. 2012; 53(2):135-9. [PubMed: 22262868]

29. Mannelli L, Yeh MM, Wang CL. A pregnant patient with hypoglycemia. Gastroenterology. 2012; 143(4):e3-4. [PubMed: 22921669]

30. Kang KM, Lee JM, Yoon JH, et al. Intravoxel incoherent motion diffusion-weighted MR imaging for characterization of focal pancreatic lesions. Radiology. 2014; 270(2):444-53. [PubMed: 24126370]

31. Jang KM, Kim SH, Lee SJ, et al. The value of gadoxetic acid-enhanced and diffusion-weighted MRI for prediction of grading of pancreatic neuroendocrine tumors. Acta Radiol. 2014; 55(2): 140-8. [PubMed: 23897307]

32. Ma X, Zhao X, Ouyang H, et al. Quantified ADC histogram analysis: a new method for differentiating mass-forming focal pancreatitis from pancreatic cancer. Acta Radiol. 2014; 55:78592. [PubMed: 24167322]

33. Schoennagel BP, Habermann CR, Roesch M, et al. Diffusion-weighted imaging of the healthy pancreas: apparent diffusion coefficient values of the normal head, body, and tail calculated from different sets of b-values. J Magn Reson Imaging. 2011; 34(4):861-5. [PubMed: 21837782]

34. Herrmann J, Schoennagel BP, Roesch M, et al. Diffusion-weighted imaging of the healthy pancreas: ADC values are age and gender dependent. J Magn Reson Imaging. 2013; 37(4):886-91. [PubMed: 23086728]

35. Balci NC, Momtahen AJ, Akduman EI, et al. Diffusion-weighted MRI of the pancreas: correlation with secretin endoscopic pancreatic function test (ePFT). Acad Radiol. 2008; 15(10):1264-8. [PubMed: 18790398]

36. Wathle GK, Tjora E, Ersland L, et al. Assessment of exocrine pancreatic function by secretinstimulated magnetic resonance cholangiopancreaticography and diffusion-weighted imaging in healthy controls. J Magn Reson Imaging. 2014; 39(2):448-54. [PubMed: 23649590]

37. Muraoka N, Uematsu H, Kimura H, et al. Apparent diffusion coefficient in pancreatic cancer: characterization and histopathological correlations. J Magn Reson Imaging. 2008; 27(6):1302-8. [PubMed: 18504750]

38. Schraibman V, Goldman SM, Ardengh JC, et al. New trends in diffusion-weighted magnetic resonance imaging as a tool in differentiation of serous cystadenoma and mucinous cystic tumor: a prospective study. Pancreatology. 2011; 11(1):43-51. [PubMed: 21412024]

39. Wang Y, Chen ZE, Yaghmai V, et al. Diffusion-weighted MR imaging in pancreatic endocrine tumors correlated with histopathologic characteristics. J Magn Reson Imaging. 2011; 33(5):10719. [PubMed: 21509863]

40. Barral M, Sebbag-Sfez D, Hoeffel C, et al. Characterization of focal pancreatic lesions using normalized apparent diffusion coefficient at 1.5-Tesla: preliminary experience. Diagn Interv Imaging. 2013; 94(6):619-27. [PubMed: 23545001]

41. Kamisawa T, Takuma K, Anjiki H, et al. Differentiation of autoimmune pancreatitis from pancreatic cancer by diffusion-weighted MRI. Am J Gastroenterol. 2010; 105(8):1870-5. [PubMed: 20216538]

42. Kartalis N, Lindholm TL, Aspelin P, et al. Diffusion-weighted magnetic resonance imaging of pancreas tumours. Eur Radiol. 2009; 19(8):1981-90. [PubMed: 19308414]

43. Klauss M, Lemke A, Grunberg K, et al. Intravoxel incoherent motion MRI for the differentiation between mass forming chronic pancreatitis and pancreatic carcinoma. Invest Radiol. 2011; 46(1): 57-63. [PubMed: 21139505]

44. Concia M, Sprinkart AM, Penner AH, et al. Diffusion-weighted magnetic resonance imaging of the pancreas: diagnostic benefit from an intravoxel incoherent motion model-based $3 \mathrm{~b}$-value analysis. Invest Radiol. 2014; 49(2):93-100. [PubMed: 24089021] 
45. Lee SS, Byun JH, Park BJ, et al. Quantitative analysis of diffusion-weighted magnetic resonance imaging of the pancreas: usefulness in characterizing solid pancreatic masses. J Magn Reson Imaging. 2008; 28(4):928-36. [PubMed: 18821618]

46. Lalwani N, Mannelli L, Ganeshan DM, et al. Uncommon pancreatic tumors and pseudo-tumors. Abdom Imaging. 2014; 40:167-80. [PubMed: 25063236]

47. Colagrande S, Belli G, Politi LS, et al. The influence of diffusion- and relaxation-related factors on signal intensity: an introductive guide to magnetic resonance diffusion-weighted imaging studies. J Comput Assist Tomogr. 2008; 32(3):463-74. [PubMed: 18520558]

48. Wang Y, Chen ZE, Nikolaidis P, et al. Diffusion-weighted magnetic resonance imaging of pancreatic adenocarcinomas: association with histopathology and tumor grade. J Magn Reson Imaging. 2011; 33(1):136-42. [PubMed: 21182131]

49. Hayano K, Miura F, Amano H, et al. Correlation of apparent diffusion coefficient measured by diffusion-weighted MRI and clinicopathologic features in pancreatic cancer patients. $\mathbf{J}$ Hepatobiliary Pancreat Sci. 2013; 20(2):243-8. [PubMed: 22311389]

50. Rosenkrantz AB, Matza BW, Sabach A, et al. Pancreatic cancer: lack of association between apparent diffusion coefficient values and adverse pathological features. Clin Radiol. 2013; 68(4):e191-7. [PubMed: 23312674]

51. Tanaka M, Fernandez-del Castillo C, Adsay V, et al. International consensus guidelines 2012 for the management of IPMN and MCN of the pancreas. Pancreatology. 2012; 12(3):183-97. [PubMed: 22687371]

52. Tanaka M. International consensus guidelines for the management of IPMN and MCN of the pancreas. Nihon Shokakibyo Gakkai Zasshi. 2007; 104(9):1338-43. [PubMed: 17827904]

53. Kang KM, Lee JM, Shin CI, et al. Added value of diffusion-weighted imaging to MR cholangiopan-creatography with unenhanced MR imaging for predicting malignancy or invasiveness of intraductal papillary mucinous neoplasm of the pancreas. J Magn Reson Imaging. 2013; 38(3):555-63. [PubMed: 23390008]

54. Fatima Z, Ichikawa T, Motosugi U, et al. Magnetic resonance diffusion-weighted imaging in the characterization of pancreatic mucinous cystic lesions. Clin Radiol. 2011; 66(2):108-11. [PubMed: 21216325]

55. Re TJ, Lemke A, Klauss M, et al. Enhancing pancreatic adenocarcinoma delineation in diffusion derived intravoxel incoherent motion f-maps through automatic vessel and duct segmentation. Magn Reson Med. 2011; 66(5):1327-32. [PubMed: 21437979]

56. Lemke A, Laun FB, Klauss M, et al. Differentiation of pancreas carcinoma from healthy pancreatic tissue using multiple b-values: comparison of apparent diffusion coefficient and intravoxel incoherent motion derived parameters. Invest Radiol. 2009; 44(12):769-75. [PubMed: 19838121]

57. Ye XH, Gao JY, Yang ZH, et al. Apparent diffusion coefficient reproducibility of the pancreas measured at different MR scanners using diffusion-weighted imaging. J Magn Reson Imaging. 2014; 40:1375-81. [PubMed: 24222019]

58. Wang CL, Chea YW, Boll DT, et al. Effect of gadolinium chelate contrast agents on diffusion weighted MR imaging of the liver, spleen, pancreas and kidney at 3 T. Eur J Radiol. 2011; 80(2):e1-7. [PubMed: 20646887]

59. Liu K, Peng W, Zhou Z. The effect of gadolinium chelate contrast agent on diffusion-weighted imaging of pancreatic ductal adenocarcinoma. Acta Radiol. 2013; 54(4):364-8. [PubMed: 23386737]

60. Beets-Tan RG, Lambregts DM, Maas M, et al. Magnetic resonance imaging for the clinical management of rectal cancer patients: recommendations from the 2012 European Society of Gastrointestinal and Abdominal Radiology (ESGAR) consensus meeting. Eur Radiol. 2013; 23(9): 2522-31. [PubMed: 23743687]

61. Soyer P, Lagadec M, Sirol M, et al. Free-breathing diffusion-weighted single-shot echo-planar MR imaging using parallel imaging (GRAPPA 2) and high b value for the detection of primary rectal adenocarcinoma. Cancer Imaging. 2010; 10:32-9. [PubMed: 20159662]

62. Curvo-Semedo L, Lambregts DM, Maas M, et al. Diffusion-weighted MRI in rectal cancer: apparent diffusion coefficient as a potential noninvasive marker of tumor aggressiveness. J Magn Reson Imaging. 2012; 35(6):1365-71. [PubMed: 22271382] 
63. Heijnen LA, Lambregts DM, Mondal D, et al. Diffusion-weighted MR imaging in primary rectal cancer staging demonstrates but does not characterise lymph nodes. Eur Radiol. 2013; 23(12): 3354-60. [PubMed: 23821022]

64. Lambregts DM, Vandecaveye V, Barbaro B, et al. Diffusion-weighted MRI for selection of complete responders after chemoradiation for locally advanced rectal cancer: a multicenter study. Ann Surg Oncol. 2011; 18(8):2224-31. [PubMed: 21347783]

65. Jang KM, Kim SH, Choi D, et al. Pathological correlation with diffusion restriction on diffusionweighted imaging in patients with pathological complete response after neoadjuvant chemoradiation therapy for locally advanced rectal cancer: preliminary results. Br J Radiol. 2012; 85(1017):e566-72. [PubMed: 22422387]

66. Song I, Kim SH, Lee SJ, et al. Value of diffusion-weighted imaging in the detection of viable tumour after neoadjuvant chemoradiation therapy in patients with locally advanced rectal cancer: comparison with T2 weighted and PET/CT imaging. Br J Radiol. 2012; 85(1013):577-86. [PubMed: 21343320]

67. Genovesi D, Filippone A, Ausili Cefaro G, et al. Diffusion-weighted magnetic resonance for prediction of response after neoadjuvant chemoradiation therapy for locally advanced rectal cancer: preliminary results of a monoinstitutional prospective study. Eur J Surg Oncol. 2013; 39(10):1071-8. [PubMed: 23953231]

68. Andreano A, Rechichi G, Rebora P, et al. MR diffusion imaging for preoperative staging of myometrial invasion in patients with endometrial cancer: a systematic review and meta-analysis. Eur Radiol. 2014; 24(6):1327-38. [PubMed: 24668009]

69. Carbone SF, Pirtoli L, Ricci V, et al. Diffusion-weighted MR volumetry for assessing the response of rectal cancer to combined radiation therapy with chemotherapy. Radiology. 2012; 263(1):311. [PubMed: 22438457]

70. Curvo-Semedo L, Lambregts DM, Maas M, et al. Rectal cancer: assessment of complete response to preoperative combined radiation therapy with chemotherapy-conventional MR volumetry versus diffusion-weighted MR imaging. Radiology. 2011; 260(3):734-43. [PubMed: 21673229]

71. Sun YS, Zhang XP, Tang L, et al. Locally advanced rectal carcinoma treated with preoperative chemotherapy and radiation therapy: preliminary analysis of diffusion-weighted MR imaging for early detection of tumor histopathologic downstaging. Radiology. 2010; 254(1):170-8. [PubMed: 20019139]

72. Jung SH, Heo SH, Kim JW, et al. Predicting response to neoadjuvant chemoradiation therapy in locally advanced rectal cancer: diffusion-weighted 3 Tesla MR imaging. J Magn Reson Imaging. 2012; 35(1):110-6. [PubMed: 21989997]

73. Lambrecht M, Vandecaveye V, De Keyzer F, et al. Value of diffusion-weighted magnetic resonance imaging for prediction and early assessment of response to neoadjuvant radiochemotherapy in rectal cancer: preliminary results. Int J Radiat Oncol Biol Phys. 2012; 82(2):863-70. [PubMed: 21398048]

74. Kim SH, Lee JY, Lee JM, et al. Apparent diffusion coefficient for evaluating tumour response to neoadjuvant chemoradiation therapy for locally advanced rectal cancer. Eur Radiol. 2011; 21(5): 987-95. [PubMed: 20978768]

75. Kim YC, Lim JS, Keum KC, et al. Comparison of diffusion-weighted MRI and MR volumetry in the evaluation of early treatment outcomes after preoperative chemoradiotherapy for locally advanced rectal cancer. J Magn Reson Imaging. 2011; 34(3):570-6. [PubMed: 21751285]

76. Kim SH, Lee JM, Hong SH, et al. Locally advanced rectal cancer: added value of diffusionweighted MR imaging in the evaluation of tumor response to neoadjuvant chemo- and radiation therapy. Radiology. 2009; 253(1):116-25. [PubMed: 19789256]

77. Park MJ, Kim SH, Lee SJ, et al. Locally advanced rectal cancer: added value of diffusion-weighted MR imaging for predicting tumor clearance of the mesorectal fascia after neoadjuvant chemotherapy and radiation therapy. Radiology. 2011; 260(3):771-80. [PubMed: 21846762]

78. Lambregts DM, Beets GL, Maas M, et al. Tumour ADC measurements in rectal cancer: effect of ROI methods on ADC values and interobserver variability. Eur Radiol. 2011; 21(12):2567-74. [PubMed: 21822946] 
79. Soyer P, Kanematsu M, Taouli B, et al. ADC normalization: a promising research track for diffusion-weighted MR imaging of the abdomen. Diagn Interv Imaging. 2013; 94(6):571-3. [PubMed: 23731499]

80. Intven M, Reerink O, Philippens ME. Repeatability of diffusion-weighted imaging in rectal cancer. J Magn Reson Imaging. 2014; 40:146-50. [PubMed: 24127172]

81. Bäuerle T, Seyler L, Münter M, et al. Diffusion-weighted imaging in rectal carcinoma patients without and after chemoradiotherapy: a comparative study with histology. Eur J Radiol. 2013; 82(3):444-52. [PubMed: 23219191]

82. Vargas HA, Akin O, Franiel T, et al. Diffusion-weighted endorectal MR imaging at 3 T for prostate cancer: tumor detection and assessment of aggressiveness. Radiology. 2011; 259(3):775-84. [PubMed: 21436085]

83. Turkbey B, Shah VP, Pang Y, et al. Is apparent diffusion coefficient associated with clinical risk scores for prostate cancers that are visible on 3-T MR images? Radiology. 2011; 258(2):488-95. [PubMed: 21177390]

84. Zhang J, Jing H, Han X, et al. Diffusion-weighted imaging of prostate cancer on 3T MR: relationship between apparent diffusion coefficient values and Ki-67 expression. Acad Radiol. 2013; 20(12):1535-41. [PubMed: 24200480]

85. Thormer G, Otto J, Reiss-Zimmermann M, et al. Diagnostic value of ADC in patients with prostate cancer: influence of the choice of b values. Eur Radiol. 2012; 22(8):1820-8. [PubMed: 22527373]

86. Tamada T, Kanomata N, Sone T, et al. High b value $\left(2,000 \mathrm{~s} / \mathrm{mm}^{2}\right)$ diffusion-weighted magnetic resonance imaging in prostate cancer at 3 Tesla: comparison with $1,000 \mathrm{~s} / \mathrm{mm}^{2}$ for tumor conspicuity and discrimination of aggressiveness. PLoS One. 2014; 9(5):e96619. [PubMed: 24802652]

87. Ueno Y, Takahashi S, Kitajima K, et al. Computed diffusion-weighted imaging using 3-T magnetic resonance imaging for prostate cancer diagnosis. Eur Radiol. 2013; 23(12):3509-16. [PubMed: 23881300]

88. Shinmoto H, Tamura C, Soga S, et al. An intravoxel incoherent motion diffusion-weighted imaging study of prostate cancer. AJR Am J Roentgenol. 2012; 199(4):W496-500. [PubMed: 22997399]

89. Dopfert J, Lemke A, Weidner A, et al. Investigation of prostate cancer using diffusion-weighted intravoxel incoherent motion imaging. Magn Reson Imaging. 2011; 29(8):1053-8. [PubMed: 21855241]

90. Kuru TH, Roethke MC, Stieltjes B, et al. Intravoxel incoherent motion (IVIM) diffusion imaging in prostate cancer - what does it add? J Comput Assist Tomogr. 2014; 38(4):558-64. [PubMed: 24733005]

91. Pang Y, Turkbey B, Bernardo M, et al. Intravoxel incoherent motion MR imaging for prostate cancer: an evaluation of perfusion fraction and diffusion coefficient derived from different b-value combinations. Magn Reson Med. 2013; 69(2):553-62. [PubMed: 22488794] 


\section{KEY POINTS}

- Intravoxel incoherent motion is a methodology to evaluate diffusionweighted imaging (DWI) with the use of multiple $b$ values, to separate the contribution of perfusion from tissue diffusion.

- $\quad$ DWI has been applied to the detection and characterization of tumors from multiple organs.

- $\quad$ DWI has been used to predict and evaluate response to treatment for a number of different tumors and treatment modalities.

- $\quad$ Reproducibility of apparent diffusion coefficients (ADC) measurements from DWI is limited by variability in imaging techniques and methods of ADC analysis. 


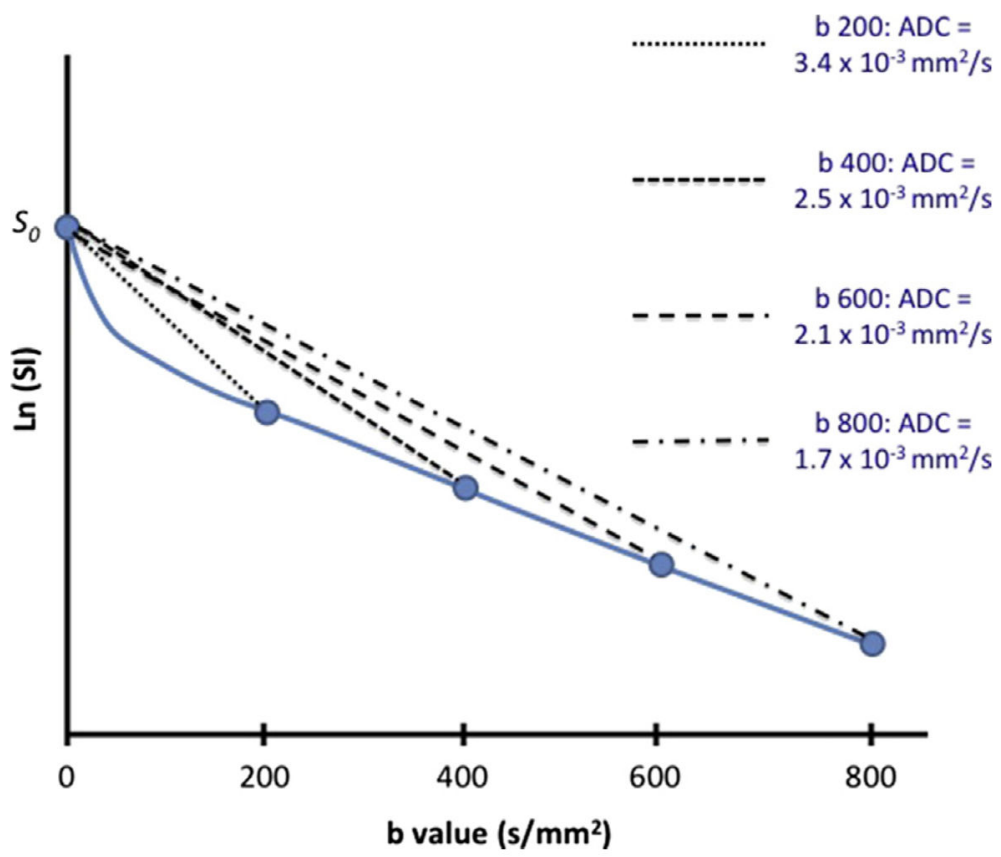

Fig. 1.

Dependence of apparent diffusion coefficients (ADC) on $b$ value used in single $b$ value diffusion-weighted image (DWI). On a logarithmic scale, the signal intensity of soft tissue is usually observed to decrease more rapidly at lower $b$ values $\left(<200 \mathrm{~s} / \mathrm{mm}^{2}\right)$ than at higher $b$ values ( $>200 \mathrm{~s} / \mathrm{mm}^{2}$ ). When DWI is limited to a single $b$ value, the calculated ADC can vary from $3.4 \times 10^{-3} \mathrm{~mm}^{2} / \mathrm{s}(b=200)$ to $1.7 \times 10^{-3} \mathrm{~mm}^{2} / \mathrm{s}(b=800)$. 


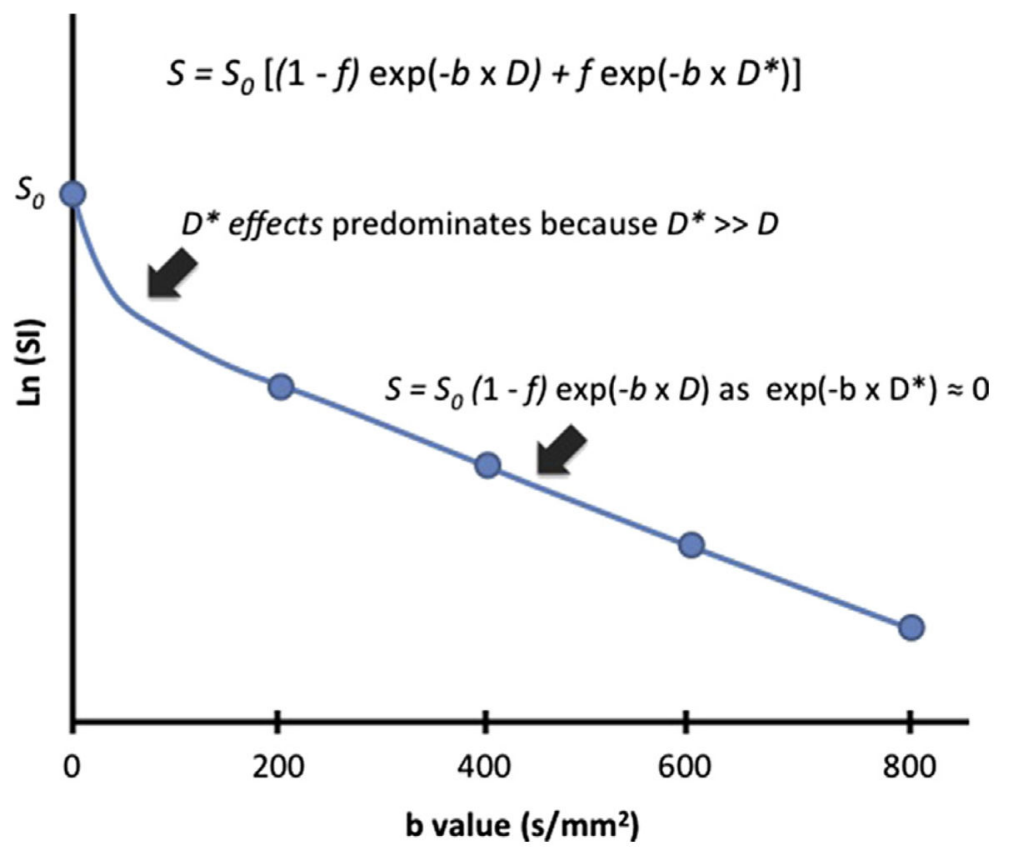

Fig. 2.

Intravoxel incoherent motion (IVIM) parameters contribution to tissue signal intensity (SI). The rapid loss in SI of soft tissue, $S$, at lower $b$ values is partly explained by the contribution of water signal from the intravascular space, which is defined by the perfusion fraction ( $f$ ) and the pseudodiffusion coefficient $D^{*}$. Because $D^{*}$ is usually several orders of magnitude larger than $D$, its contribution approaches zero as $b$ increases above 100 to $200 \mathrm{~s} / \mathrm{mm}^{2}$. Thus, at higher $b$ values ( $>200 \mathrm{~s} / \mathrm{mm}^{2}$ ), a monoexponential behavior is observed from the extravascular fraction of moving water molecules $(1-f)$, which is governed by the tissue diffusion coefficient $D$. 

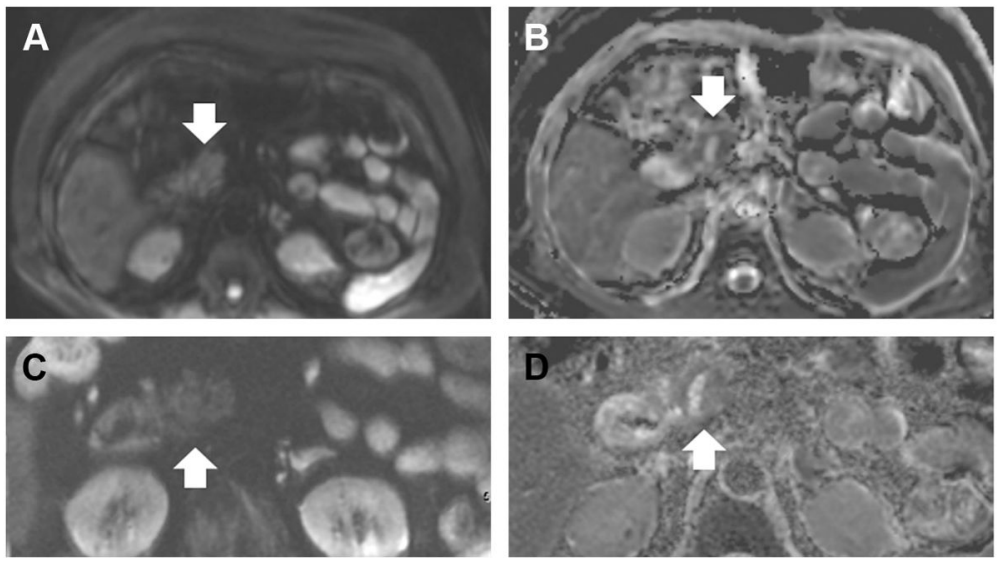

Fig. 3.

Diffusion-weighted image (DWI) of the pancreas with large and small fields of view (FOV). Large FOV $(A)$ and small FOV DWI $(C)$ were obtained of the pancreas, with corresponding apparent diffusion coefficient maps $(B, D)$. Finer details can be seen in the pancreatic head (arrow) with the small FOV DWI, which has a higher in plane resolution of $1.6 \times 1.6 \mathrm{~mm}^{2}$ (compared with $3.1 \times 3.1 \mathrm{~mm}^{2}$ ). 

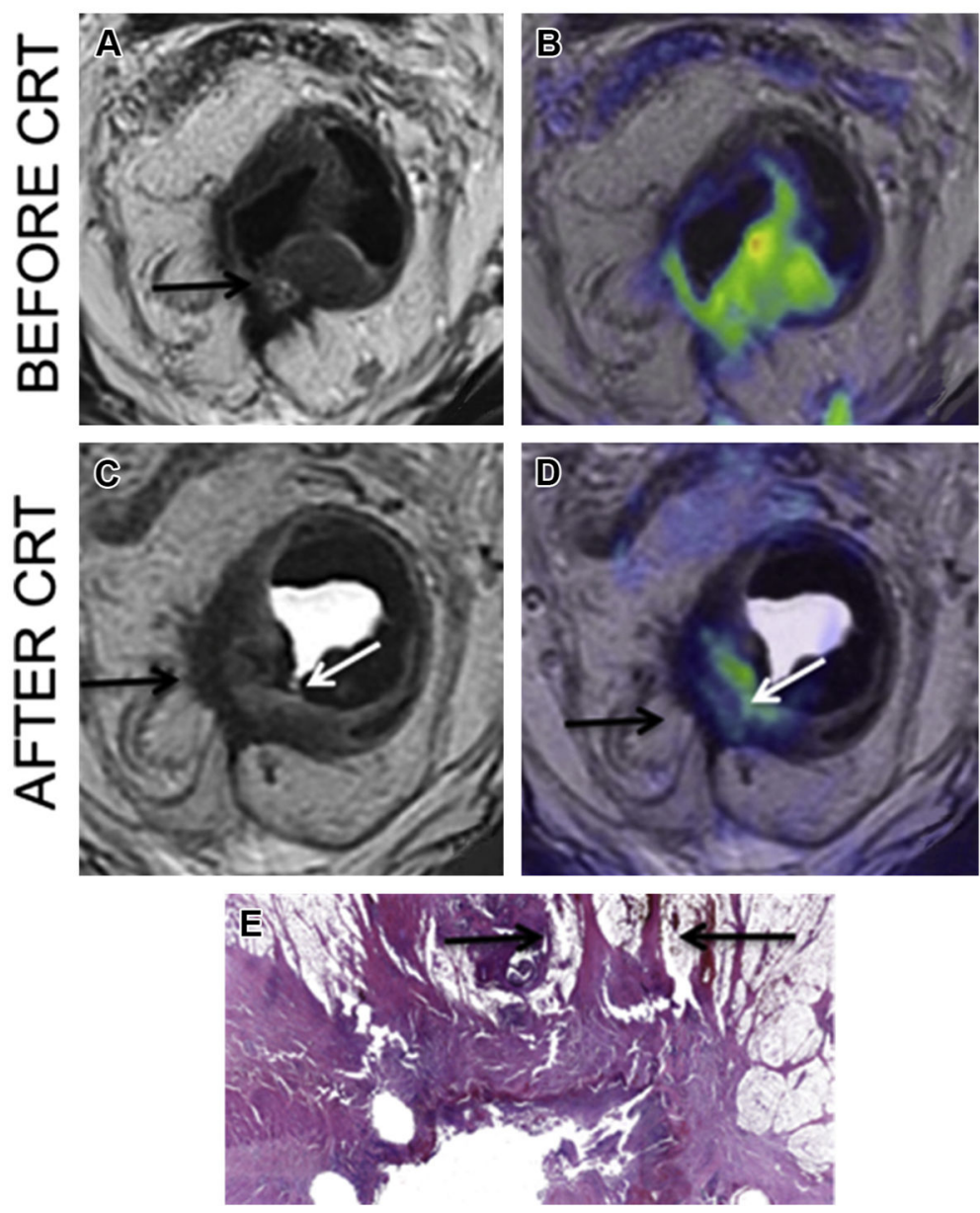

Fig. 4.

Rectal cancer diffusion-weighted image (DWI). Before neoadjuvant chemoradiotherapy (CRT), T2WI sequence ( $A$; arrow) and fused T2WI/DWI $(B)$ image demonstrate T3c tumor extending thorough the mesorectal fascia. After CRT, the T2WI $(C)$ demonstrate residual tumor (white arrow) but also T2 hypointense area (black arrow) in the muscularis propria with speculation extending thorough the mesorectal fascia. This could either represent fibrotic scar or residual tumor. Adding DWI to T2WI $(D)$ enables to distinction of residual tumor (white arrow) from fibrotic scar (black arrow) confirmed on pathology (E) (arrow, original magnification, $\times 4$; stain, hematoxylineosin). 


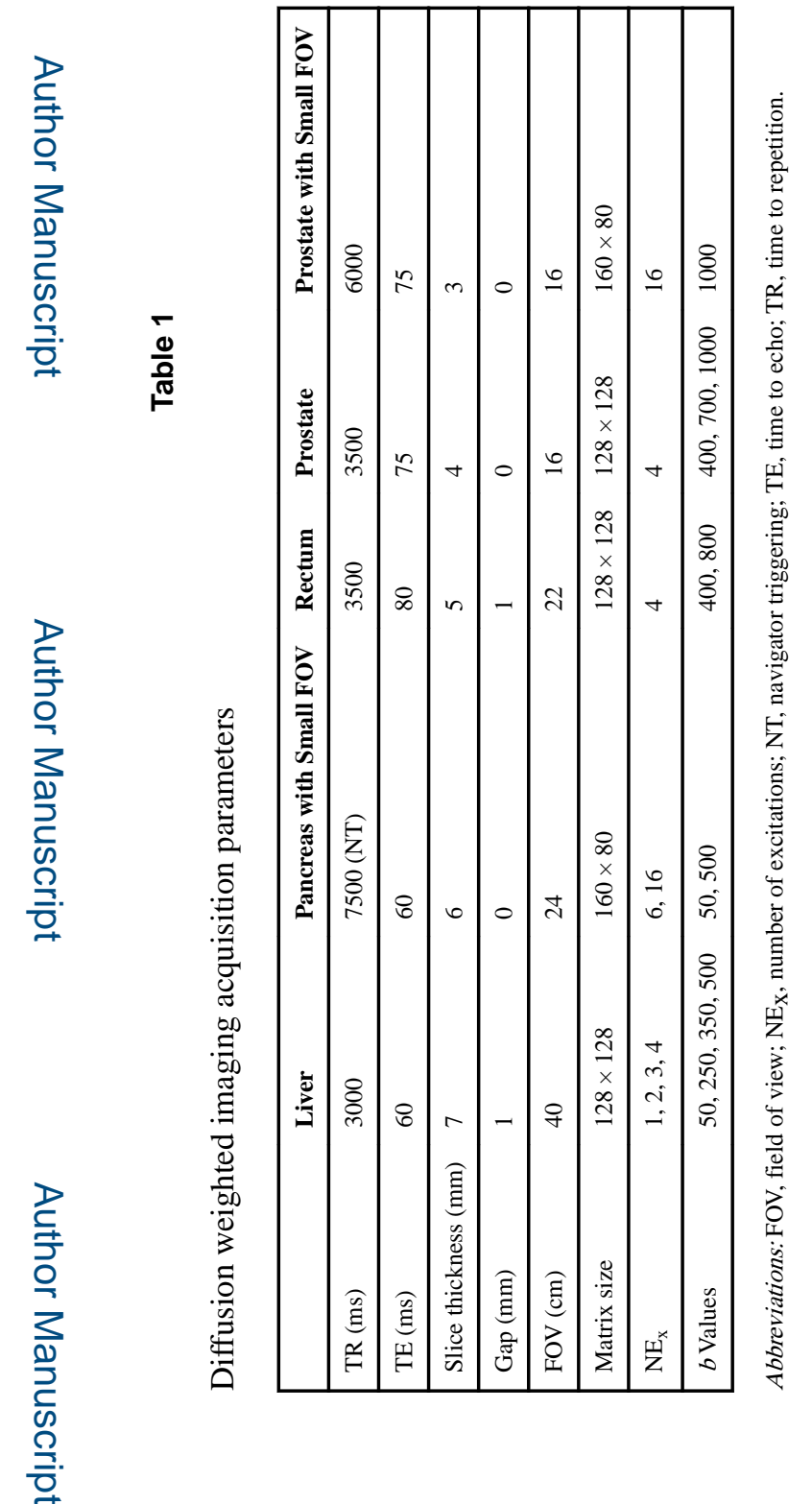

Radiol Clin North Am. Author manuscript; available in PMC 2016 August 10. 